\title{
Prostatic Cyst with Bladder Outlet Obstruction Symptoms. Case Report
}

\author{
Alemayehu Tegegne Tefera, María E Suárez Marcillán. \\ Department of Urology at St Paul's Hospital Millennium Medical College \\ Correspondence to:- Alemayehu Tegegne Tefera, Email: \\ alett2005@gmail.com \\ https://dx.doi.org/10.4314/ecajs.v22i1.17
}

The prostatic cysts are uncommon lesions usually detected incidentally. The incidence is reported as less than 1\% most often occurs as small \& asymptomatic lesions located medially in the gland, when they get a big size causes different lower urinary tract symptoms. Only 5\% are symptomatic. The symptoms depend on the size \& location of the lesion. Minimal access surgery (endoscopic) is recommended for its treatment. We present a case with the diagnosis of Prostatic cyst at the bladder neck treated with transurethral resection of the lesion. Histopathological investigation revealed benign prostatic cyst. At six months of follow up the patient remains free of symptoms.

Key words: Prostatic Cyst, Bladder outlet obstruction, transrectal ultrasound (TRUS), Transurethral Resection of the Prostate (TURP).

\section{Introduction}

The prostatic cysts are extremely rare, frequently diagnosed incidentally with an incidence of less than $1 \%$. The uses of imaging studies have made cyst incidences increased. Usually they are small and asymptomatic located in the middle of the gland. When they have a big size causes different lower urinary tract symptoms. Only $5 \%$ are symptomatic. ${ }^{1}$ Dik $\mathrm{P}$, et al. ${ }^{2}$ reported a series of transrectal ultrasound on 704 patients with symptoms of bladder outlet obstruction or lower urinary tract symptoms and prostatic cyst was found in 34 (5\%). These might include painful ejaculation, hemospermia, infertility, urinary tract infections, chronic pelvic pain and prostatitis-like syndrome symptoms, but can rarely result in obstructive and irritative urinary tract symptoms if located close to the bladder neck or posterior urethra. ${ }^{1,3}$

Prostatic cysts are diagnosed incidentally during un ultrasound examination for any other indication and are classified based on cyst location, shape, and embryogenic origin, interconnection with prostatic urethra or seminal vesicle, and sperm presence in the cyst. ${ }^{1}$, 3 The increasing use of transrectal ultrasound (TRUS) has resulted in the discovery of incidental prostatic cysts. Galosi et al. classified 6 distinct cyst types based on TRUS and pathological features, including midline cyst, cyst of the ejaculatory ducts, cyst of the parenchyma, multiple cysts, complicated cyst, cystic tumor, and cyst secondary to other diseases. ${ }^{4}$ Kilinc, Tambo, Nghlem, clasifiedthe prostatic cysts lessons in congenital (mullerian duct and utricle) or acquired (ejaculatory duct cysts). 1, 3,5 The Mullerian duct cysts and Prostatic utricle cysts are considered two separate clinical entities. The first arise from 
remnants of the mullerian duct and the second as their name implies, are due to dilatation of the prostatic utricle. These two conditions can be very difficult or impossible to distinguish from clinically, and sonographically. Mullerian duct cysts usually occur in the 3rd and 4 th decades of life. Utricle cysts are most often detected earlier in life. ${ }^{5}$

The mullerians cysts are considered a remnant of the duct of Müller. Commonly are presented with irritative urinary symptoms, hematuria, suprapubic or rectal pain and palpable suprapubic mass, and sonographically they extend cephalic and lateral to the midline of the prostate gland. ${ }^{1,3,5}$

It has been reported the association of urogenital anomalies like the unilateral renal agenesis, undescended testis and hypospadias with the presence of cysts of the utricle that tend to be smaller in size and rarely spread outside the prostate to be considered a dilation of the utricle, it gives some symptoms, on post voiding ultrasound the cystic image is seen in the middle part of the gland and the distinctive clinical feature is post micturation dribblimg of urine. Mullerian duct cysts have no such associations. This difference is the reason for the belief that utricle cysts have a different embryologic origin than mullerian duct cysts. Endoscopic contrasted study of the cyst confirms the diagnosis of the cyst. $\mathbf{1 , 3 , 5 , 6 , 7}$

The ejaculatory cysts are even rarer, raises the theory that are caused by a congenital obstruction of the duct or by secondary inflammation. It is asymptomatic and is diagnosed accidentally during investigation for some other pathology.

When the cysts are large in size are accompanied of perineal pain, dysuria, hemospermia and pain during ejaculation. When it has a big size the ultrasound shows as cystic lesion at the ejaculatory duct, the small cysts are generally seen as cystic lesions in the central and lateral areaof the base of the gland at the level of verummontanum. It is associated with the presence of stones to that level. The aspiration of the cyst may report the presence of sperm if testicular function is normal. After endoscopic contrast injection of the prostatic cyst can be appreciated dilated seminal vesicle on the same side. ${ }^{5,6}$

The cystic degeneration of benign prostate enlargement often occurs with relative frequency and commonly located in the area of transition. These cysts are small and rarely give symptoms; The result of cyst puncture is frequently starch bodies, hemorrhagic fluid, infarction or necrosis of the hyperplasic nodule ${ }^{5}$

The prostate abscesses are usually complications of prostate infections (bacterial prostatitis) being the E. coli the more frequent causal germ. It has a high incidence in patients in the 5th and 6th decade of life, although its incidence has increased at young ages related to individual sexual behaviors. The patients present with the typical signs and symptoms of prostatitis; the ultrasound finding is focally enlarged prostate, hypoechoic or anechoic lesion, with irregular walls, internal echoes or partitions. These lesions are generally observed toward the midline of the gland. 5, 6,7 The clinical presentation improvement and the response to the antibiotic treatment confirms the diagnosis. 
Other entities are reported even it is rare are parasitic prostate cysts (eg, Echinococcus cyst, bilharzial cyst) and cystic carcinoma. 1, 3, 6, 7. In the literature, recommended many treatments of prostatic cysts include TRUSG guided drainage or esclerotherapy, endoscopic transurethral resection or transurethral incision with endoscopic urethrotomy, Holmium: YAG laser for the marsupialization of the cyst wall, and in some cases even open surgery. ${ }^{\mathbf{1}, 2}$, $3,4,6$

We present a case with a diagnosis of prostatic cyst. We will present:-the symptoms, investigations performed, treatment, follow-up and a literature review.

\section{Case Report}

A 54-year-old male patient was admitted to our hospital with a history of chronic suprapubic pain of more than 6 months, and nocturia. Genital examination, external meatus, and digital rectal examination of the patient revealed normal findings. PSA $0.44 \mathrm{ng} / \mathrm{ml}$. Abdominal ultrasonography revealed normal prostate size $(3.6 \mathrm{~cm} \times 3.4 \mathrm{~cm})$, volume $22 \mathrm{ml}$ with midline prostatic cysts $(2.4 \mathrm{~cm} \times 1.9 \mathrm{~cm})$ and high post voiding residual $(156 \mathrm{ml})$ (Figure 1). His International Prostate Symptom Score (IPSS) was 20, and his quality of life (QoL) score was 4. The cyst was homogeneous on Pelvic CT, but in MRI wasn't visualized. Under spinal anesthesia, Cystoscopic examination performed and found normal prostate size and a cystic mass at the $6 o^{\prime}$ clock position on the bladder neck, which appeared to be obstructing the bladder outlet. (Figure 2) The mass was unroofed using the transurethral resection loop. Subsequently, a $16 \mathrm{Fr}$ Foley catheter was inserted at the end of the intervention. On the 1st postoperative day, the urethral catheter was removed. The histopathological examination revealed a benign prostatic cyst. During the 6-month follow-up period, no complications were observed and symptoms disappeared. The anterograde ejaculation was preserved.

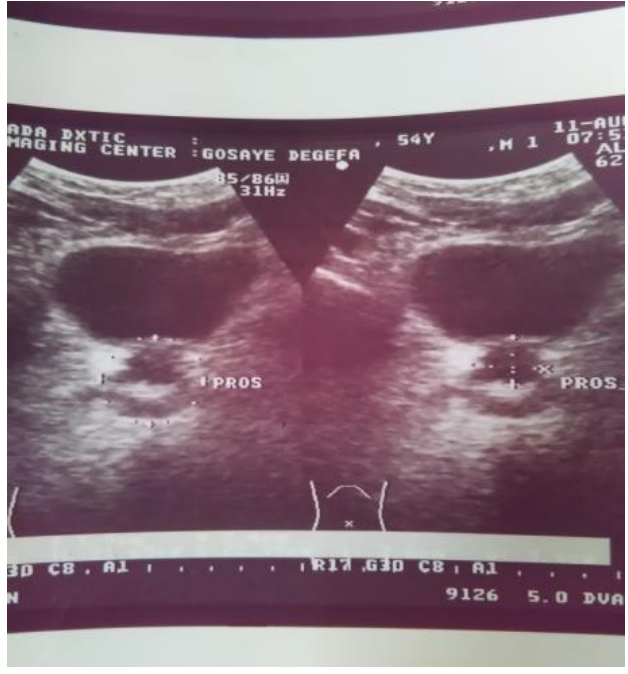

Figure 1. Abdominal ultrasonography findings: midline prostatic cysts $(2.4 \mathrm{~cm} \mathrm{x}$ $1.9 \mathrm{~cm})$.

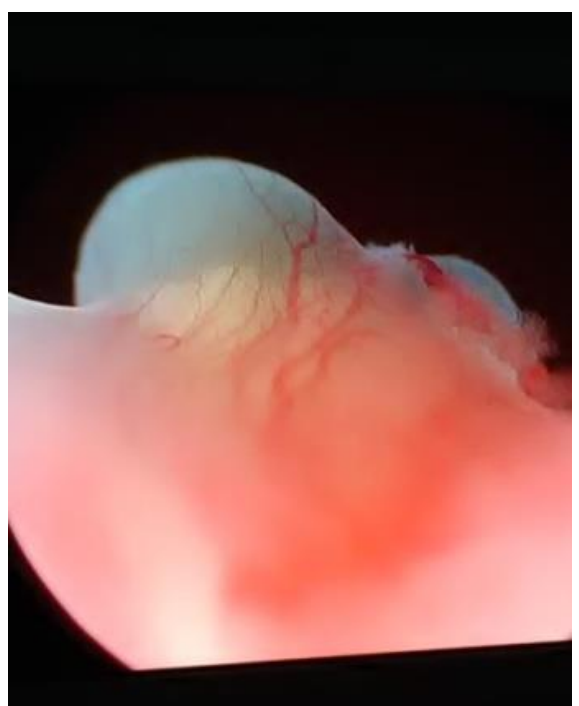

Figure 2. Cystoscopic finding: cystic mass at the 6 o' clock position on the bladder neck. 


\section{Discussion.}

The increasing use of the imaging studies as an abdominal ultrasound and transrectal ultrasound (TRUS) have increased of incidental finding of prostatic cysts. The reports of prostatic cysts has increased every time in the literatures. $1,2,3,4$

Galosi et al. reported that midline cysts are observed by TRUS in $9.8 \%$ of caseslocated posteriorly, ${ }^{4}$ similar as our case of a prostatic cyst located at 6 'clock position on the bladder neck. Kilinc, et al, reported a 26 -year-old male patient with a history of chronic suprapubic pain for more than 2 years, and painful ejaculation. Transrectal ultrasound (TRUSG) revealed a midline prostatic cyst with approximately $10 \times 12 \mathrm{~mm}$ diameter. The pelvic MRI verified the same and midline prostatic cyst. Minimally invasive endoscopic intervention using holmium was done: YAG laser was considered to treat long term lower urinary tract symptoms in order to minimize potential harm inflicted on the urogenital tract and future sexuality of the young patient. During 3- and 6-month follow-up periods, no complications were observed. Suprapubic pain and painful ejaculation disappeared. ${ }^{1}$

Tambo, et al, reported two cases of symptomatic prostatic cysts arising around the bladder neck causing bladder obstruction for long time (5 - 10 years) and the quality of life was affected. The patients were investigated with Uroflowmetry (obstructive patron) and abdominal ultrasonography (high post voiding residual). The abdominal ultrasonography revealed prostatic cysts arising around the bladder neck. The cyst was homogeneous on pelvic CT and MRI. With flexible cystoscope showed a smooth bulge anteriorly at the proximal prostatic urethra. The cysts were at the 11 o' clock positions on the bladder neck, appeared to be obstructing the bladder outlet, did not show prostatic hypertrophy. The mass were unroofed using the trans urethral resection loop. The histopathological examination revealed benign prostatic cysts. The anterograde ejaculations were preserved and postoperatively the symptoms were resolved. ${ }^{3}$ The highest number of patients with symptomatic prostatic cysts have been reported by Tambo, et al with 34 patients. The symptoms included obstructive urinary tract symptoms (40\%), urinary retention (33\%), urodynia (9\%), and infertility (6\%). Symptomatic prostatic cysts were seen in relatively young patients and usually measures $3.6 \mathrm{~cm} .^{3}$

Joo-Yong et al. reported the first case of a prostatic cyst in Korea in a 41-year-old man presented with LUTS. The prostatic cyst was located anteriorly and acted like a checking valve during urination. His International Prostate Symptom Score (IPSS) was 20, and his quality of life (QoL) score was 4 . Uroflowmetry showed that the peak flow rate was $9 \mathrm{ml} / \mathrm{s}$ (136 $\mathrm{ml}$ voided volume) and the volume of residual urine was more than $200 \mathrm{ml}$; a digital rectal examination revealed a normal prostate. Urine cytology did not suggest malignancy, and his serum PSA level was $3.12 \mathrm{ng} / \mathrm{ml}$. The patient was treated with transurethral resection of the cyst, and the obstructive symptoms successfully improved. ${ }^{8}$

Tambo and Joo-Yong reported cases of prostatic cysts arising around the bladder neck similar to our case, ${ }^{3}, 8$ but the localization of the cyst of our case was posterior. The 
retention cysts of the prostate are resulting from the obstruction of the glandular acinis causing its dilation. Usually appear between the 5th and 6th decade of life. Usually cause no symptoms but when they are located at the level of the bladder neck can cause obstructive symptoms by the closure of the same. Mostly they are located toward the transitional zone and measure between 1-2 cm in diameter or less. May be one way indistinguishable from cystic changes of the BPH. ${ }^{5}$ Taketoshi et al reported a 54-year-old man with dysuria. Transabdominal ultrasonography revealed a multilocular cyst at the neck of the bladder, and a cystoscopy revealed bladder neck obstruction. The initial treatment was tamsulosin and abdominal magnetic resonance imaging (MRI) and the cyst disappeared. After 2 years the patient came again with difficulty of urination and transurethral resection of the cyst was performed. Histopathological examination reported a retention cyst. ${ }^{9}$ Hiroshi et al reported a 34-year-old man presented a prostatic retention cyst around the bladder neck causing prostatitis-like symptoms. The investigations (TRUS, MRI and Cystoscopy) revealed a projecting prostatic cyst which occupied the bladder outlet and seemed to cause the symptoms. Transurethral resection of the cyst was performed and the symptoms were markedly improved. Histopathologically, the cyst was retention cyst of the prostate. ${ }^{10}$

Dell reported a 28 year old young man with obstructive and irritative voiding disorders caused by a prostatic cyst, located in the anterior and left lateral lobe of the prostate gland. The prostatic cyst was incised and marsupialized by transurethral resection. At postoperative follow up observed an increase in Qmax with no residual urine and negative urine culture. The patient showed no retrograde ejaculation or erectile dysfunction. ${ }^{11}$ on the other handDik $P$, et al. did in their series ultrasound guided transurethral marsupialization of the cyst through prostatic floor incision in 18 patients without complication. ${ }^{2}$

The recommended treatment for intraurethral prostatic cyst is transurethral resection. This treatment can resolve obstructive urinary tract symptoms and preserve ejaculation in the absence of the prostatic hypertrophy ${ }^{3,6,8,9,10,11}$. The complications are rare. Young patients need special care to preserve antegrade ejaculation and future fertility. In order to minimize the risk of complications is recommended the use of lower-Fr endoscopic instrument with minimal trauma to the bladder neck and urethra. ${ }^{1,3,8,9,10,11}$

The malignancy natures of the prostatic cysts are less frequent. The lower male genitourinary tract is an extremely rare location for adenoid cystic carcinoma. Frankel and Craig in 1974 reported the first case with a diagnosis of adenoid cystic carcinoma of the prostate as an extremely rare variety of prostate adenocarcinoma 6, 7 the tumor is histologically identical to adenoid cystic carcinoma of the salivary gland and breast. In these sites has favorable prognosis. The patient was treated with transurethral resection of the tumor with negative long-term follow-up. ${ }^{6}$

Since 1974 for more than a decade only four well-documented adenoid cystic carcinomas has been reported in detail in the literatures where In 1988 two new cases were reported for Young, et al. in men of 60 and 68 years of age with urinary tract obstruction of long duration. The patients were treated with transurethral resection. When diagnoses were 
confirmed one patient had a pelvic lymph node dissection recovered 26 nodes, all of which were negative for tumor, and a radical prostatectomy was then performed. The patient is well six years after operation. The other patient was treated with transurethral resection and radiation and is well eight months after operation ${ }^{7}$. Adenoid cystic carcinoma is considered for many authors as a subtype of prostatic adenocarcinoma ${ }^{6,7}$. This can be a justification of the therapeutic treatment follow up in these cases because evidence suggests that the adenoid cystic-like tumor has an excellent prognosis on the basis of the limited experience to date ${ }^{6}$.

\section{Conclusion}

Minimal Access surgical techniques (endoscopic) continue to be preferable treatment option for prostatic cysts with excellent results and minimal risk of complications.

\section{References}

1. Kilinc M, Goger Y.E, Piskin M, et al. Midline Prostatic Cyst Marsupialization Using Holmium Laser. Case Reports in Urology. 2015. Article ID 797061 http://dx.doi.org/10.1155/2015/797061

2. Dik P, Lock TM, Schrier BP, et al. Transurethral marsupialization of medial prostatic cysts in patients with prostatitis-like symptoms. J Urol. 1996 Apr; 155 (4): 1301 - 4. PMID: 8632560.

3. Tambo M, Okegawa T, Nutahara K, et al. Prostatic cyst arising around the bladder neck-cause of bladder outlet obstruction: Two case reports. Act Urol Jpn. 2007; 53: 401 - 407. http://hdl.handle.net/2433/71422

4. Galosi $A B$, Montironi $R$, Fabiani $A$, et al. Cystic lesions of the prostate gland: an ultrasound classification with pathological correlation. J Urol. 2009; 181: 647-657.

5. Nghlem et al. Cystic Lesions of the Prostate. Radiographic. 1990; 10 (4): 635 - 650.

6. kenneth F, John R. C. Adenoid Cystic Carcinoma of the Prostate. Am. J. Clin. Pathol. 1974; 62: 639-645. http://ajcp.oxfordjournals.org/

7. Young R, Frierson $\mathrm{H}$, et al. Adenoid Cystic-Like Tumor of the Prostate Gland. A Report of Two Cases and Review of the Literature. A.J.C.P. 1988; 89 (1): 49-56. http://ajcp.oxfordjournals.org/

8. Joo-Yong L, Dong-Hyuk K, Hee-Young $P$, et al. An Anteriorly Positioned Midline Prostatic Cyst Resulting in Lower Urinary Tract Symptoms. Int Neurourol J. 2010 Aug; 14(2): 125-129. doi: 10.5213/inj. 2010.14.2.125 PMCID: PMC2989466.

9. Taketoshi K, Hiroki A, Nobukazu M, et al. Retention Cyst of the Prostate in a Patient with Urinary Difficulty. Act Urologic Japonica. 2012; 58 (3): 173 - 175. http://hdl.handle.net/2433/154881

10. Hiroshi S, Norihide K, Hitoshi S, Satoshi H. Prostatic Retention Cyst around the bladder neck: A case report. 泌尿器科紀要 2009; 55 (9): 583 - 586 . http://hdl.handle.net/2433/85364

11. Dell A. Disorders of bladder depletion in a young man because of a prostatic cyst: a case report. Arch Ital Urol Androl. 2012 Mar; 84 (1): 44 - 6. 